\title{
The New Method of Double Fold Operation Using Hybrid Technique: Combined Incisional and Non-incisional Method
}

\author{
Nam Seok Park \\ Point Plastic Surgery, Seoul, Korea
}

No potential conflict of interest relevant to this article was reported.
Background Double-fold operation is one of the most common types of surgery in Korea and can be divided into two major surgical techniques, incisional and non-incisional methods. Each method has its own advantages and disadvantages. Therefore, the author describes a new procedure to take advantage of both methods.

Methods This procedure was performed in 127 consecutive patients (106 primary cases, 21 secondary cases) whose ages ranged from 19 to 65 years. First, a double-fold was made using the non-incisional method, and then excessive fat and redundant skin were removed using the incisional method. Fat was removed if necessary. For patients with blepharoptosis, before removing the redundant skin, this condition was corrected with the non-incisional Müller tucking ptosis method.

Results Of the 127 patients, 122 patients were satisfied with the result. Edema and scarring were less than with the incisional method, and the postoperative result was easier to predict than with the secondary double-fold operation. Three cases had shallow eyelid lines, and one case had a complete loss of the fold. One secondary case patient had asymmetry that was corrected with revision.

Conclusions Because satisfactory results can't be achieved easily with the double-fold operation, a challenge for plastic surgeons is choosing the best method for the doublefold operation, especially in secondary cases. This new method that uses a hybrid technique that combines the incisional and non-incisional methods may be an effective choice when performing the double-fold operation in both primary and secondary cases.

Keywords Blepharoptosis, Blepharoplasty, Buried suture technique

\section{INTRODUCTION}

Double-fold operation is the most common type of aesthetic surgical procedure in Korea. However, due to its popularity, double-fold operation is associated with certain complications. An incision-type double-fold operation may cause dissatisfaction in new patients because too many variations are available for them to consider, in-

Received: Feb 2, 2015 Revised: Apr 2, 2015 Accepted: Apr 3, 2015 Correspondence: Nam Seok Park Point Plastic Surgery, 308 Apgujeong-ro, Gangnam-gu, Seoul 135-896, Korea. E-mail: mdnspark2@naver.com

Copyright @ 2015 The Korean Society for Aesthetic Plastic Surgery.

This is an Open Access article distributed under the terms of the Creative Commons Attribution Non-Commercial License (http://creativecommons.org/licenses/by-nc/3.0/) which permits unrestricted non-commercial use, distribution, and reproduction in any medium, provided the original work is properly cited. $\quad \boldsymbol{w} w \boldsymbol{w} . \boldsymbol{e}$-aaps.org cluding formation of a line without a coincident cutting line, asymmetry, and the difficulty in setting an appropriate eyelid length. Furthermore, reoperation can be problematic for not only new patients but also for quite experienced plastic surgeons due to the difficulty in the design of a new line, possible edema during the surgery, error due to hematoma, and the uncertain formation of eyelid lines.

Therefore, to solve the above problems, the author designed a "first-buried then-incision" method. First, formation of the eyelid line, which is the final result of all blepharoplasty procedures, is performed; other procedures are performed later. The author obtained a satisfactory result with the procedure described above, which uses both the burying technique and the incision technique to ensure a rapid procedure and high reliability in primary and secondary cases. 


\section{METHODS}

From January 2012 to March 2014, this procedure was performed in 127 consecutive patients (106 primary cases, 21 secondary cases) whose ages ranged from 19 to 65 years with a mean age of 38 years. The group included 12 men and 115 women. They all underwent the surgery for the sole purpose of cosmetic improvement. For patients who underwent reoperation, 13 had deep eyelid lines, five had asymmetric balance, and three had shallow eyelids and excess scarring.

According to an interview with the patients, we set the appropriate length of the line in the sitting position, and in cases of reoperation, a thorough discussion was made with patients to understand the length of the line suggested by the surgeons and limited amendment of the surgery.

The ideal length of the eyelid line and the upper margin of the line was designed with a gentian-violet wooden pen, and depended on the amount of redundant skin or scar tissue to be excised. The operation was performed in a supine position. Administration of propofol for sedation was performed in case of patient anxiety or with a combination of any other type of surgery such as rhinoplasty. A mixture of $2 \%$ lidocaine and 1:100,000 epinephrine was injected into the hypoderm. A tagging suture was made with 6-0 black silk below the predefined position. The conjunctiva layer was exposed by turning the upper eyelid inside out using a cotton swab, and then the same mixture of local anesthesia was injected. At the level of the designed eyelid line, $1 \mathrm{~mm}$-sized small slits were made using a No. 11 blade. Through the slit, a penetrating No. 7-0 double needle blue nylon was inserted in the order of skin-tarsal plate-conjunctiva-skin and stitched up using the interrupted non-incision double-fold method (Fig. 1). In cases of reoperation, scar tissue

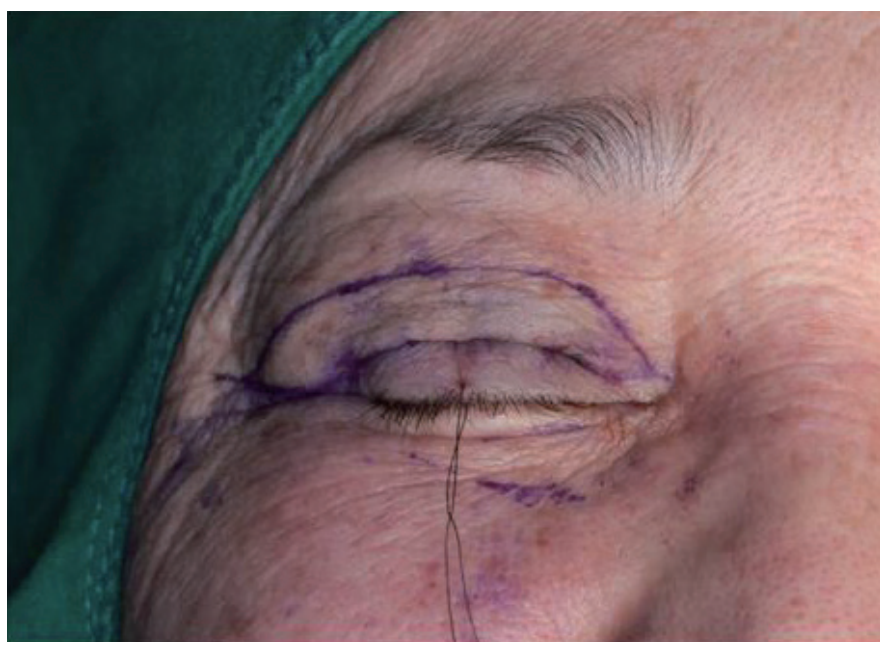

Fig. 1. Surgical marking. The desired double-fold line and upper margin, depending on the amount of skin excision, is drawn. Through the small slits, the double-fold line is made using the non-incisional method. formation was seen around the previous double-fold line. In such cases, through the slits, the scar tissue was separated from the un-
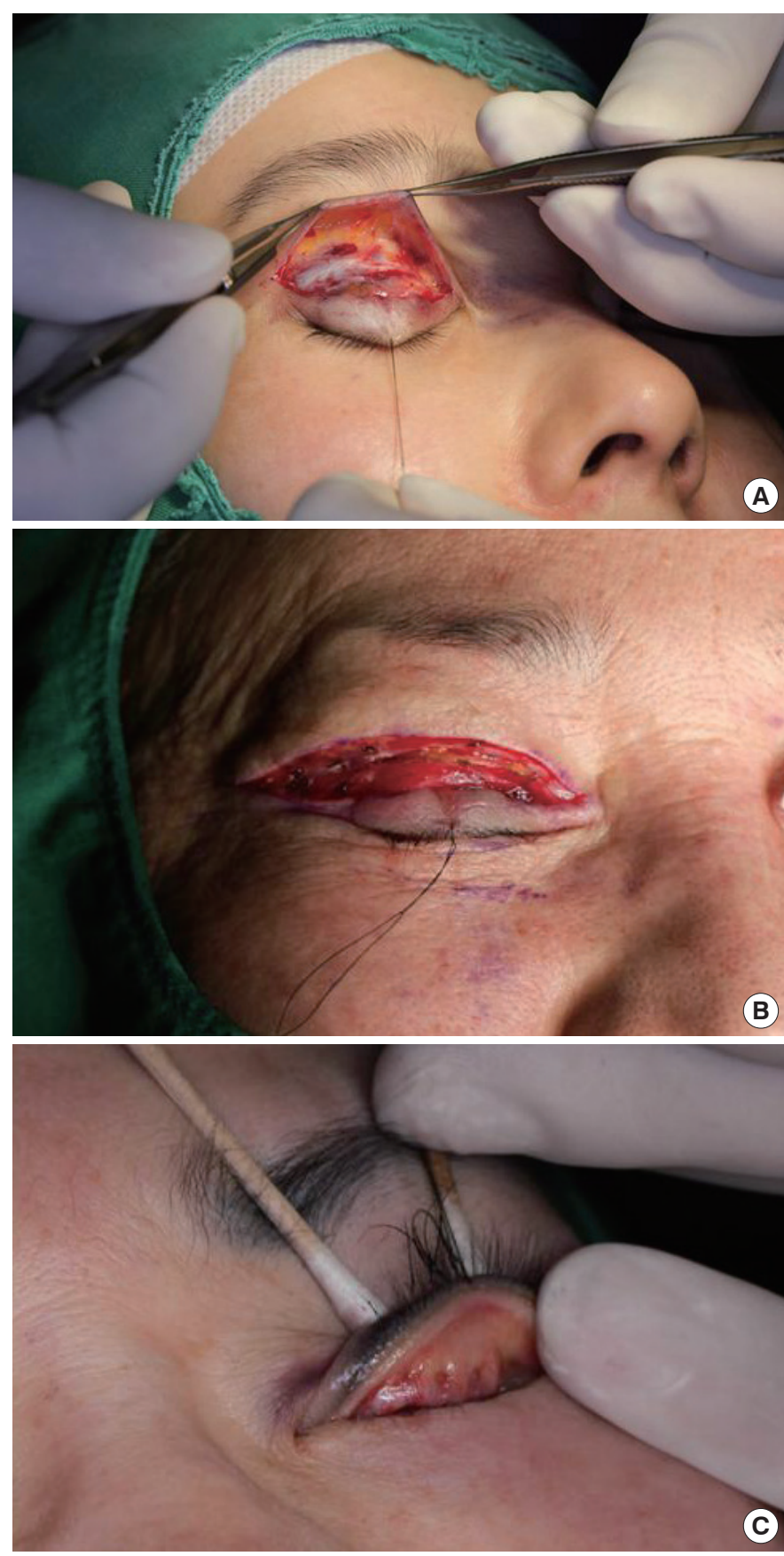

Fig. 2. Operative procedure. (A) Scar tissue dissection. When the lower incision line is cut to remove the scar tissue, the scar tissue is easily separated from the normal tissue with dissection with small curved iris scissors through the small slits. (B) Skin excision. Redundant skin is removed using the incisional method after making the double-fold line using the non-incisional method. (C) Non-incision Müller tucking ptosis method. In patients with blepharoptosis, the non-incisional Müller tucking ptosis method is performed before redundant skin or scar tissue is excised. 
derlying normal tissue using small curved iris scissors. A doublefold line was then made using the method described above (Fig. 2A).

After formation of a line with the non-incisional double-fold method, the lower boundary of the remaining skin or the scar tissue, which included small incision slits and the upper designed line,

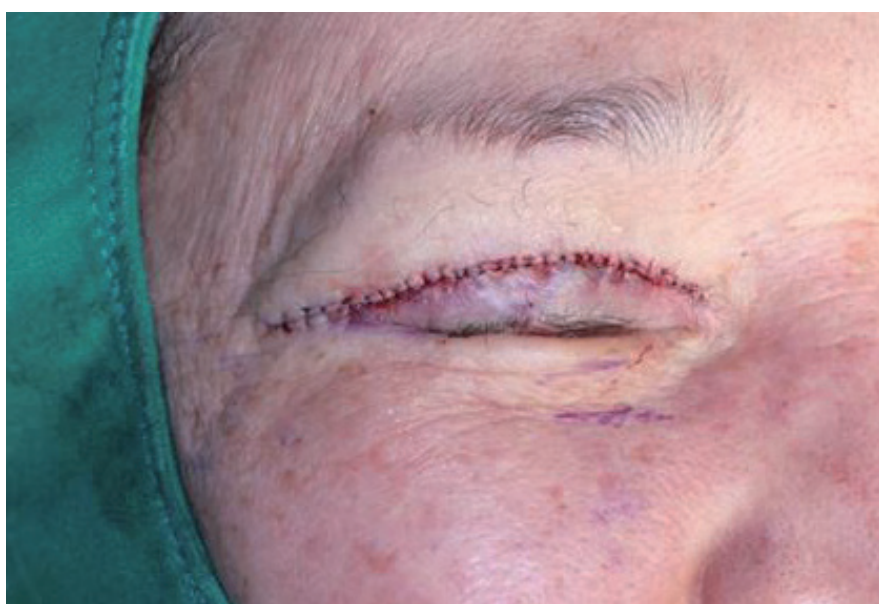

Fig. 3. Immediate postoperative photographic finding after skin closure.
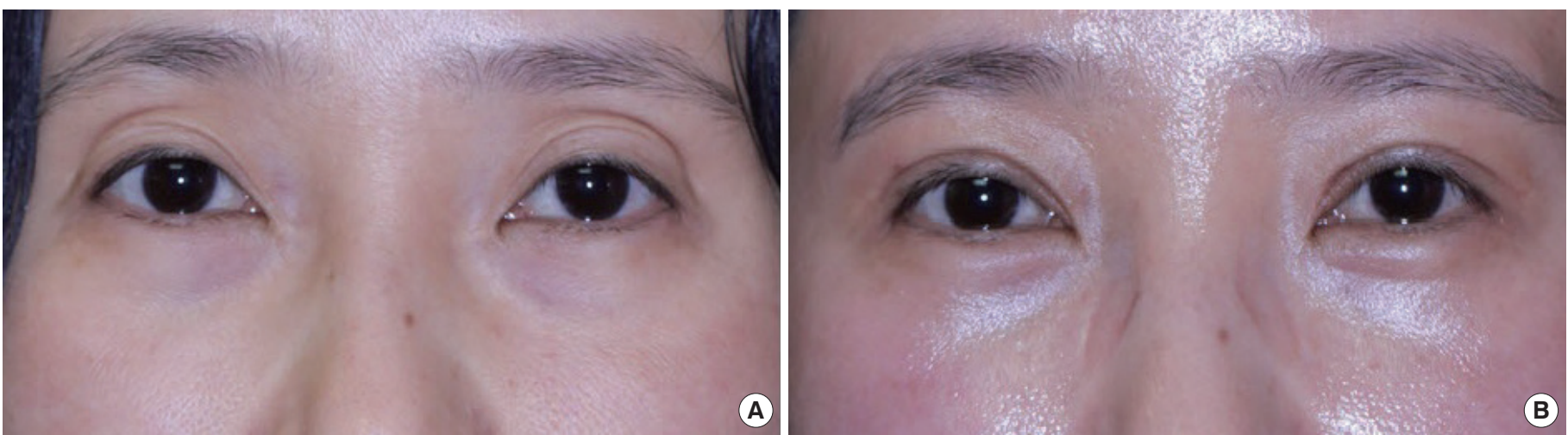

Fig. 4. Case 1. (A) Preoperative photographic finding. (B) Postoperative photographic finding.
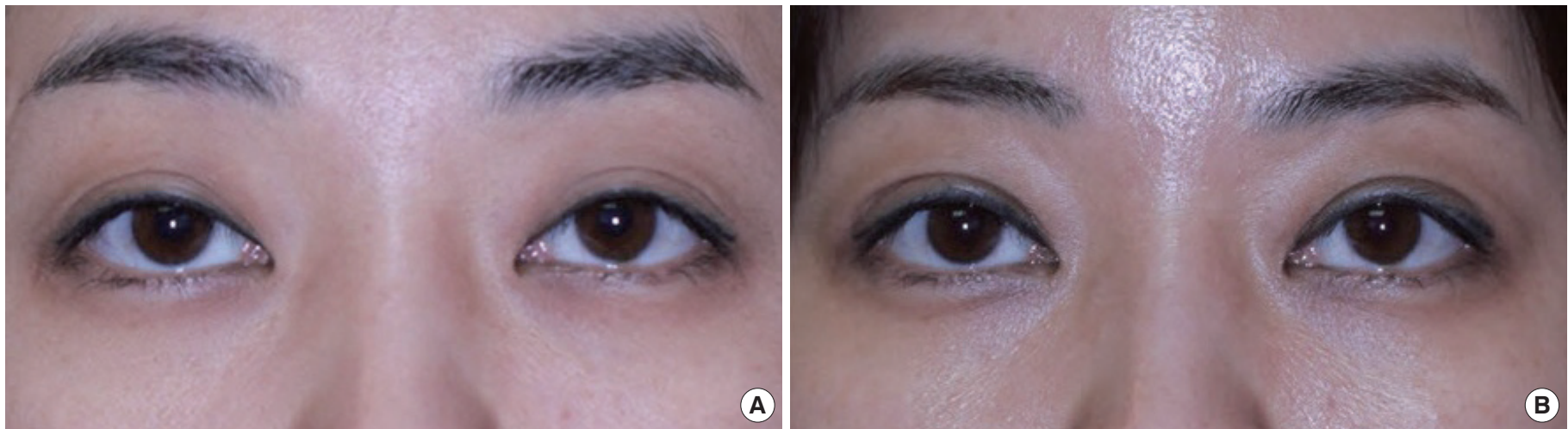

Fig. 5. Case 2. (A) Preoperative photographic finding. (B) Postoperative photographic finding. 
one side.

The cicatrix after the surgery was very similar to that following the sole incision method. Edema was present for 5 to 7 days postoperatively. According to the author's experience, however, the edema was less intense than that resulting from the incision method surgery. In addition, in cases of reoperation, the edema was less than that resulting from sole incision surgery. At 10 to 14 days after the surgery, an eyelid line of appropriate length had formed (Fig. 4 and 5).

The follow-up time was 3 to 26 months, with an average of 9 months. Except for one case, no sign of loosening or complete loss of the double fold was seen after the surgery.

\section{DISCUSSION}

Double-fold operation is the most commonly performed plastic surgery in oriental countries including Korea [1]. Recently, plastic surgery has become widespread. The awareness of it by the population has increased, and it has become more accepted. Anatomically, Asians have a large amount of fat on their eyelids. Because the fiber connecting the levator palpebrae superioris muscle does not reach the inner eyelid of the corium, statistically, about $30 \%$ to $60 \%$ of the population has single eyelids $[2,3]$.

The double-fold operation can be classified into three major methods, incision, partial incision, and burying method, each with its own pros and cons [4].The incision method involves soft tissue removal and then induces cicatricial adhesion with the succession of tissue layers to eradicate the large amount of excess fat. However, the advantage of this technique can be reduced by swelling and bleeding during the operation that makes fastening difficult. Furthermore, in the case of reoperation, deciding where to set the line is difficult and requires a lot of experience and confidence in predicting the formation of lines.

In 1896, Mikamo first introduced the burying technique, which is now favored by most surgeons because the surgery is rapid and easy to perform, the recovery time is short, and scarring does not occur after surgery [5]. Nevertheless, this method has a limitation because removing the excess tissue is impossible, and this method cannot be used except in cases of line disappearance. In addition, in 1989, Baek et al. [6] observed the disappearance of eyelid lines in $2.9 \%$ of patients after surgery. Homma et al. [7] reported the disappearance of eyelid lines in $1 \%$ of patients during the first year after surgery, and in $3.43 \%$ of patients during 5 years after surgery. This disappearance of eyelid lines after the burying technique is a limi- tation of this method.

Unlike the traditional method of line fixation after skin removal, the author reversed the procedure. At first, a double-fold line was made using the burying technique, and then fat and redundant skin were removed with aso-called "first-burying then-incision" method. This method produced the expected result after surgery. Moreover, this method created a well-balanced symmetry and allowed a firm creation of the line with adequate adhesion. Also, in cases of reoperation, this technique allows surgeons to make accurate, new lines as expected. These features are the positive characteristics for those who first undergo this surgery.

Although the double-fold operation is the most common plastic surgery operation, satisfactory results are not achieved easily. In addition, no definitive evidence exists showing that the outcome is better with incisional compared to the non-incisional method, because each method has advantages and disadvantages. Thus, a challenge exists for plastic surgeons to choose the best method for double-fold operation, especially in cases of secondary surgery. The author suggests that the "first-burying then-incision" method will be very effective in primary and secondary cases of double-fold operation.

\section{PATIENT CONSENT}

Patients provided written consent for the use of their images.

\section{REFERENCES}

1. Boo-Chai K. Some aspects of plastic (cosmetic) surgery in orientals. Br J Plast Surg 1969;22:60-9.

2. Kim YK, Kwon JD, Oh KS. Double eyelid operation with three tiny incisions. J Korean Soc Plast Reconstr Surg 2000;27:195-8.

3. Hiraga Y. The double eyelid operation and augmentation rhinoplasty in the Oriental patient. Clin Plast Surg 1980;7:553-67.

4. Chung WJ, Lee YJ, Baek RM, et al. Partial incision double fold operation using debulking method. J Korean Soc Aesthetic Plast Surg 2004; 10:1-6.

5. Shirakabe Y, Kinugasa T, Kawata M, et al. The double-eyelid operation in Japan: its evolution as related to cultural changes. Ann Plast Surg 1985;15:224-41.

6. Baek SM, Kim SS, Tokunaga S, et al. Oriental blepharoplasty: singlestitch, nonincision technique. Plast Reconstr Surg 1989;83:236-42.

7. Homma K, Mutou Y, Mutou H, et al. Intradermal stitch blepharoplasty for orientals: does It disappear? Aesthetic Plast Surg 2000;24:289-91. 